\title{
An Atom-probe Tomographic Study of Kinetic Pathways of Retention Excesses and Depletions at Gamma(F.C.C.)/gamma-prime (L12) Interfaces in a Ni-Al-Cr-Re Superalloy
}

Sung-Il Baik and David Seidman

Northwestern University, Evanston, Illinois, United States

The nucleation, growth and coarsening kinetics of coherent gamma-prime-(L12)-precipitates in a quaternary $\mathrm{Ni}-10 \mathrm{Al}-8.5 \mathrm{Cr}-2 \mathrm{Re}$ (at.\%) alloy, (1), aged at $700{ }^{\circ} \mathrm{C}$ from 0 to $1024 \mathrm{~h}$, are studied utilizing atom-probe tomography (APT) and transmission electron microscopy (TEM). The temporal evolutions of the concentration profiles yield retention excesses and depletions at gamma(FCC)/gamma-primeinterfaces, which are discussed in detail. The compositional trajectories are plotted in a quaternary phase diagram, which is displayed in a tetrahedron whose apexes are equal to 100 at.\% $\mathrm{Ni}, \mathrm{Al}, \mathrm{Cr}$ and $\mathrm{Re}$, thereby yielding a three-dimensional picture of the compositional evolutions of the gamma-prime(L12)precipitates and gamma(FCC)-matrix phase. The gamma-prime(L12)-phase is nucleated in the gammaprime (L12) phase-field and it commences evolving with a curvilinear composition trajectory, which corresponds to the nucleation and growth regimes of the gamma-prime(L12)-precipitates. This curvilinear composition trajectory becomes linear when stationary-coarsening commences and it continues as a vector until it reaches the solvus-surface between the gamma-prime(L12)-phase-field and the [gammaprime(L12) plus gamma(FCC)]-phase-field. Alternatively, the gamma(FCC)phase commences with a mean composition of Ni-10Al-8.5Cr-2Re (at.\%) in the [gamma-prime(L12) plus gamma(FCC)]-phasefield it begins with a curvilinear composition trajectory, corresponding to the nucleation and growth of the gamma(FCC)-phase. This trajectory becomes linear when stationary coarsening occurs and it stops on a conjugate solvus-surface, which is between the [gamma-prime(L12) plus gamma(FCC)]-phase-field and the gamma(FCC)-phase field. A straight-line (a vector) can then be drawn between these two endpoints, which is a tie line for this mean composition. The stationary coarsening regime is analyzed in terms of the Philippe-Voorhees (P-V) coarsening model, which includes the thermodynamics of the gamma(FCC)matrix phase and a diffusion tensor that includes off-diagonal terms, implying that solvent-solute terms are contributing to the fluxes that exist in this quaternary alloy. When the off-diagonal terms are neglected then solvent-solute terms are absent, which can yield a misleading picture of the temporal evolution of the gamma-prime(L12)-precipitate phase.

\section{References}

1. S.-I. Baik, Z. Mao, C.E. Campbell, C. Zhang, B. Zhou, R.D. Noebe, D.N. Seidman, “An Atom-Probe Tomographic Study of the Compositional Trajectories During gamma (fcc)/gamma-prime (L12) PhaseSeparation in a Ni-Al-Cr-Re Superalloy," Cornell University Condensed Matter, 2019. https://arxiv.org/abs/1904.13035 
https://doi.org/10.1017/S1431927620020358 Published online by Cambridge University Press 